\title{
Las dimensiones de personalidad como predictores de los comportamientos de ciudadanía organizacional
}

\author{
Alicia Omar \\ Consejo Nacional de Investigaciones Científicas y Técnicas, Argentina \\ Hugo Uribe Delgado \\ Universidad Nacional de Rosario, Argentina
}

\begin{abstract}
Resumo
As dimensões de personalidade como preditores dos comportamentos de cidadania organizacional. O comportamento de cidadania organizacional (CCO) se refere às condutas benéficas para a organização que não são contratualmente estipuladas, nem formalmente recompensadas. O objetivo do presente estudo foi explorar o conjunto de dimensões de personalidade como preditores dos CCO de ajuda e de voz. A amostra foi constituída por 335 empregados argentinos, que preencheram uma folha de dados sociodemográficos, o questionário de Personalidade de Eysenck e as escalas de CCO de ajuda e de voz desenvolvidas por Van Dyne e seus colaboradores. Análises de regressão stepwise indicaram que a tendência a extroversão constitui o melhor preditor dos CCO de voz, enquanto que os CCO de ajuda foram melhor explicados pela estabilidade emocional. Os resultados proporcionaram apoio parcial às relações hipotetizadas entre psicoticismo e CCO de voz, uma vez que as tendências não-psicóticas se revelaram bons preditores dos CCO de voz somente entre as mulheres. Os resultados dão base para elaboração de uma agenda para futuras investigações na área.
\end{abstract}

Palavras-chave: comportamentos de cidadania organizacional; comportamentos de ajuda; comportamentos de voz; dimensões eysenckianas de personalidade

\begin{abstract}
Personality dimensions as predictors of organizational citizenship behavior. Organizational Citizenship Behavior (OCB) involves discretionary behavior, not required or formally rewarded, that has positive consequences for the organization. The main goal of this study was to examine the role of personality dimensions as predictors of employee's engagement in help and voice OCB. Sample was integrated by 335 Argentinean employees, which were asked to complete a battery of questionnaires including demographic items, Eysenck Personality Questionnaire and Help and Voice OCB scales developed by Van Dyne and his colleagues. Stepwise regression analysis revealed that extraversion was the best predictor of the voice behavior, and emotional stability was the best predictor when help behavior was the criterion. Results partially supported the hypothetical relationship between psicoticism and voice behavior, since non psychotic tendencies only emerged as predictors of voice among females. New research agenda designed to further our understanding of personality-OCB linkages is proposed.
\end{abstract}

Keywords: organizational citizenship behavior; help behavior; voice behavior; Eysenckian's personality dimensions

$\mathrm{D}$ esde que Organ (1988) acuñara el término de Comportamiento de Ciudadanía Organizacional (CCO), para referirse a todo comportamiento discrecional, que sobrepasa las expectativas formalmente requeridas para el desempeño de un determinado rol y que resulta beneficioso para las organizaciones, se viene registrando un rápido crecimiento de las investigaciones desarrolladas con el objetivo de evaluar la pertinencia empírica de tales comportamientos. Análisis críticos de este cuerpo de conocimientos indican que se trata de un comportamiento organizacional al que se le han atribuido diferentes denominaciones; gran número de definiciones; carácter multidimensional y que los mayores esfuerzos han estado orientados hacia la identificación de sus antecedentes.

En cuanto a las denominaciones que ha ido recibiendo, es dable observar que hasta mediados de la década de los 
años 1990, el CCO también era identificado como un comportamiento organizacional espontáneo (George \& Brief, 1992); comportamiento organizacional prosocial (Puffer, 1987) o como un comportamiento extra papel (Bateman \& Organ, 1983). Si bien los límites entre comportamientos extra e intra papel suelen ser interpretados de diferente manera por parte de los empleados (Morrison, 1994), ya que mientras algunos pueden creer que un comportamiento dado es un CCO, otros, que definen más ampliamente sus responsabilidades laborales, pueden considerar la misma tarea como un comportamiento inherente a su rol. Una muestra elocuente de tal distorsión perceptiva lo constituyen los hallazgos del reciente trabajo de Vey y Campbell (2004), quienes encontraron que frente a un listado de ítems orientados a explorar CCO y comportamientos intra papel, el 85\% de los participantes categorizó como intra papel a 17 de los 30 ítems de CCO.

En cuanto a las definiciones, aunque la literatura científica de los últimos 15 años presenta un muestrario de intentos al respecto, en la actualidad se observa cierto acuerdo por caracterizar a los CCO en base a las siguientes cuatro notas distintivas: a) el comportamiento debe ser intencional, o sea, tiene que ser el resultado de una decisión deliberada para ejecutarlo; b) tiene que ser voluntario, o sea, no puede ser formalmente prescripto y recompensado, ni formalmente castigado en el caso de su no ocurrencia; c) tiene que ser desinteresado, o sea, básicamente orientado a beneficiar a alguien o alguna cosa, más que al propio actor y d) tiene que ser percibido de manera positiva, ya sea por el propio actor ya sea por el observador (LePine, Erez \& Jonson, 2002; Van Dyne, Cummings, \& Parks, 1995).

Si bien la multidimensionalidad del constructo no está en discusión, aún persiste el debate en relación al número de componentes y su correspondiente operacionalización. En sus postulaciones iniciales, Organ (1988) propuso un modelo integrado por cinco dimensiones: ayuda, cortesía, espíritu deportivo, rectitud y virtud cívica. Modelo que fue expandido mediante la inclusión de dos nuevas dimensiones, armonía interpersonal y protección de los bienes de la empresa, por parte de Mac Kenzie, Podsakoff y Fetter (1993) y Podsakoff, MacKenzie y Hui (1993). Posteriormente, y como resultado de la integración de perspectivas filosóficas, políticas e históricas, Van Dyne, Graham y Dienesch (1994), propusieron un modelo conformado por las categorías de obediencia, lealtad y virtud cívica, las que, según ellos, conformarían el síndrome de ciudadanía activa, por el cual los trabajadores se comprometen a una participación responsable en su lugar de trabajo. Morrison (1994) criticó esta nueva conceptualización, subrayando que la dimensión de virtud cívica o civismo estaba referida a los comportamientos de los ciudadanos que tienen consecuencias para el Estado, más que a comportamientos que tienen consecuencias en las relaciones interpersonales. Situación que condujo a Siqueira (1995, citado por Porto \& Tamayo, 2003) a resaltar la inadecuación del término ciudadanía organizacional y sugerir el empleo del término civismo organizacional.
Con el propósito de aclarar el panorama conceptual, y manteniendo la denominación de CCO, Van Dyne et al. (1995) propusieron una taxonomía basada en dos dimensiones contrastantes: comportamiento promotor y prohibitivo, por un lado, y comportamiento afiliativo y desafiador, por el otro. El comportamiento promotor es proactivo, estimula y hace que las cosas ocurran; el prohibitivo es preventivo, incluye la protección de individuos con menor poder o evita la ocurrencia de conductas inapropiadas o no éticas. El comportamiento afiliativo es interpersonal y cooperativo, fortalece las relaciones y está orientado hacia el otro; el comportamiento desafiador enfatiza ideas y problemas, está orientado a los cambios y puede perjudicar las relaciones interpersonales. La clasificación de los diferentes tipos de CCO que viene siendo más frecuentemente estudiada por los especialistas, surge a partir de la combinación cruzada de estas dimensiones. En este sentido, por ejemplo, un CCO con énfasis en la ayuda y en la cooperación sería un comportamiento afiliativo y promotor; prácticas ilegales o inmorales ejecutadas por los empleados a sabiendas de los empleadores (whistle-blowing behavior) y prácticas contestatarias que tienden a cambiar el status quo organizacional (principled organizational dissent), serían comportamientos prohibitivos y desafiadores.

La investigación sobre CCO tuvo como objeto inicial de interés los comportamientos de ayuda o colaborativos, aunque últimamente la atención de los investigadores también se está orientando al estudio de los comportamientos de $v o z$ (voice behavior) (LePine \& Van Dyne, 1998). Los CCO de ayuda se traducen en colaboraciones frente a un problema $\mathrm{o}$ una tarea relevante para la organización, tales como orientar a los nuevos compañeros, reemplazar a los ausentes, compartir la sobrecarga de trabajo y similares. El estudio del CCO de voz, aunque es uno de los más recientes en el conjunto de los CCO, actualmente es considerado como uno de los más importantes para el éxito de una organización por el énfasis que pone sobre las cuestiones de flexibilidad, innovación y desarrollo continuo. La manifestación libre de opiniones y el desafío constructivo al status quo con el objetivo de mejorar, y no simplemente de criticar, pueden contribuir no sólo al éxito del grupo, sino también, y principalmente, al éxito de las organizaciones (Van Dyne, Ang, \& Botero, 2003). A efecto del presente trabajo serán tratados con mayor detalle los comportamientos de ayuda y de voz, ambos vistos CCO promotores: el de ayuda, es el más tradicional y frecuentemente estudiado en la literatura, corresponde a uno de los aspectos centrales del concepto de ciudadanía organizacional y tiene cuño afiliativo; el de voz, de carácter desafiador, es uno de los que menos atención ha recibido en la literatura, tal vez por haber sido introducido más recientemente.

Aunque ayuda y voz sean ambos comportamientos promotores y proactivos e indiquen que los individuos que se identifican con ellos están satisfechos con sus organizaciones, se distinguen entre sí en varios aspectos. En primer lugar, porque el primero tiene por objetivo preservar o desarrollar las relaciones interpersonales, mientras que el se- 
gundo puede perturbarlas. De esta manera, por ejemplo, cuando un miembro de un grupo trae una sugerencia constructiva e innovadora que implica cambios en un procedimiento estandarizado con vistas a la mejora del flujo de trabajo, su idea puede incomodar a los demás miembros del grupo (LePine \& Van Dyne, 1998). En segundo lugar, porque la ayuda implica aceptación y orientación hacia el presente, mientras que la voz sugiere cambio y se orienta hacia el futuro, lo que puede ser, respectivamente, traducido por las expresiones “está OK” y “podría ser mejor” (Van Dyne \& LePine, 1998).

Si bien la taxonomía de Van Dyne y cols. (1995) ha hecho una importante contribución a la organización teórica de este campo de estudio, no se acompaña de datos empíricos acerca de su validez. Desde la perspectiva empírica, los estudios recién se inician, por lo que todavía son escasos los trabajos que se proponen evaluar la validez de constructo y la validez predictiva de los CCO de ayuda y voz. Direccionando esfuerzos en este sentido, Van Dyne y LePine (1998) adaptaron de Organ y Konovski (1989) la escala de ayuda, de siete ítems, mientras que Van Dyne, Graham y Dienesch (1994) desarrollaron una escala de voz, con seis ítems. Ambas escalas demostraron su validez predictiva y de constructo una vez que fueron sometidas a análisis factoriales confirmatorios y de regresión jerárquica (Van Dyne \& LePine, 1998 y LePine \& Van Dyne, 1998).

La reconceptualización de Van Dyne et al. (1995; 1998), puede ser considerada como una nueva fuente teórica, importante para el desarrollo de la investigación futura de este fenómeno, en la medida que especifica algunos potenciales antecedentes y consecuentes de los CCO. Entre los antecedentes, los autores examinan la posible influencia conjunta de factores personales y factores situacionales relativos al ambiente de trabajo y, entre los consecuentes, las implicancias simultáneas a nivel individual y grupal-organizacional. Desde entonces el estudio de los CCO ha despertado un creciente interés entre los investigadores de las organizaciones y suscitado el desarrollo de numerosos estudios destinados a profundizar el conocimiento de sus antecedentes. Sin duda alguna, la relación entre las percepciones de justicia y el CCO ha sido una de las más investigadas últimamente (Ehrhart, 2004; Kickul, Lester \& Finkl, 2002; Rego, 2000; Siqueira, Gomide Jr. \& Oliveira, 2002), apoyada en la noción de intercambio social, o sea, en el supuesto que si una organización trata a sus empleados justamente ellos probablemente retribuirán con esfuerzos extras para el bien de la empresa. Sin embargo, otras variables organizacionales están siendo consideradas como antecedentes privilegiados de los CCO, tales como satisfacción laboral (Yoon \& Suh, 2003), involucramiento (Diedendorff, Brown, Kamin, \& Lord, 2002), compromiso (Becker \& Kernan, 2003; Pearce \& Herbik, 2004), valores organizacionales (Van Dyne, Vandewalle, Kostova, Latham, \& Cummings, 2000; Tamayo, 1998), percepción de apoyo por parte del supervisor (Hui, Lee, \& Rousseau, 2004), contrato psicológico (Coyle-Shapiro \& Kessler, 2003; Van Dyne \& Pierce, 2004), confianza en el supervisor (Aryee, Budhwar, \& Chen, 2002) y similares variables organizacionales.

\section{La personalidad como predictor de los CCO}

Aunque inicialmente Smith, Organ y Near (1983) sugirieron que los comportamientos extra papel podrían ser una manifestación de disposiciones amplias hacia la conducta prosocial, muchos de los estudios pioneros sobre CCO y diferencias individuales no han logrado demostrar relaciones significativas (George, 1991; Konovsky \& Organ, 1996). Reiterando sus presunciones, en 1994 Organ señala que habría que analizar si la lógica por la que se predice una asociación entre actitudes laborales y CCO (o sea, en base a algunas disposiciones subyacentes), también sirve para predecir relaciones entre personalidad y CCO. En esta misma línea argumental, Organ y Ryan (1995) remarcan que no sería apropiado minimizar el rol de las disposiciones con respecto a los CCO, ya que es posible que los estudios preliminares hayan examinado sólo un conjunto limitado de variables disposicionales, cuyos efectos podrían haber sido atenuados por “situaciones fuertes” (Mischel, 1977), típicas de muchos entornos organizacionales, donde las demandas propias de los incentivos externos limitan la variabilidad conductual.

Podría considerase entonces que los CCO, por su propia naturaleza, representan conductas que ocurren en "situaciones débiles" - aquellas exentas de incentivos externos apremiantes para la conducta. No obstante, si los CCO son medidos a través del grado en que los participantes responden habitualmente a tales situaciones, podría esperase que tales grados capturaran operacionalmente las tendencias a través de muchas instancias y oportunidades para responder de tal manera (Organ, 1994). En resumen, se podría esperar en los CCO el tipo de performance que es atribuible a la personalidad, por lo que podrían actuar como un criterio frente al cual se podría establecer la candidatura de la personalidad - o al menos de ciertas dimensiones de personalidad -, como una variable independiente para explicar las contribuciones individuales al éxito organizacional.

En términos generales, los resultados de los estudios orientados a predecir CCO a partir de dimensiones de personalidad parecieran ser decepcionantes. Esto podría deberse, al menos, a dos cuestiones puntuales: al empleo de algunas variables de personalidad de los Big Five y de ciertos tipos de CCO. Con respecto al primer punto, habría que destacar que ciertas dimensiones de los Big Five comparten el espacio semántico de ciertos CCO, produciendo un cierto solapamiento. En este sentido, los constructos Big Five denominados Rectitud (que puede ser empíricamente definido a través de adjetivos tales como: puntual, cuidadoso, disciplinado, confiable y describe a las personas que generan respeto y tratamientos favorables) y Conformidad (que satura en el opuesto de los pares adjetivales: mezquino-generoso y rudocortés y describe a las personas que se llevan bien con todos los que las rodean), incluyen una cantidad de facetas o rasgos típicos de CCO tales como cortesía, obediencia o virtud 
cívica. Por lo que más que actuar como predictores, se asimilarían a algunos de los CCO habitualmente explorados en la literatura de finales de la década de los años 1990. A su vez, en los pocos estudios que han analizado las relaciones entre personalidad y CCO (Barrick, Mount, \& Judge, 2003; Motowidlo \& Van Scotter; 1994; Smith, Organ, \& Near, 1983), la variable Extraversión prácticamente no explica porcentajes apreciables de la varianza de los CCO. No obstante, con la introducción del CCO de voz por parte de Van Dyne y sus colaboradores, sería esperable que la dimensión Extraversión emergiera como un predictor idóneo de tal CCO. Con respecto a la dimensión Neuroticismo, se ha sugerido (Staw, Bell, \& Clausen, 1986) que la afectividad positiva sería la principal candidata entre las variables disposicionales. Si la afectividad positiva generalmente elicita conductas prosociales, entonces la afectividad positiva como una variable disposicional podría predecir la tendencia a ejecutar CCO de ayuda (Fisher, 2002), como reflejo de la frecuencia con que las personas, a través del tiempo y de las situaciones, se comprometen con tales conductas. De manera similar, si los afectos negativos suprimen el comportamiento prosocial, la afectividad negativa, como un rasgo, debería estar asociada con una menor frecuencia de CCO manifiestos de ayuda.

Consecuentemente, en base a las consideraciones precedentes, el propósito del presente estudio se orienta a verificar empíricamente las potencialidades de las dimensiones eysenckianas de personalidad (Eysenck, 1970, 1985, 1991) como predictores de los CCO de ayuda y de voz. En el modelo tridimensional de personalidad de Eysenck, la dimensión afectiva o Neuroticismo indica predisposiciones emocionales, mientras que la dimensión temperamental o Extraversión es conceptualizada como un indicador de sociabilidad, optimismo y asertividad. Posiblemente, el sujeto extravertido, por su mayor predisposición a establecer y mantener contactos interpersonales, tendría una mayor tendencia a involucrarse en CCO de voz, mientras que el sujeto emocionalmente estable (o no neurótico), posiblemente protagonizaría una mayor cantidad de CCO de ayuda, como reflejo de la ecuanimidad, formalidad y amabilidad que lo caracteriza, a diferencia de los sujetos emocionalmente inestables (neuróticos) caracterizados por rasgos tales como susceptibilidad, ansiedad y pusilanimidad. En base a tales suposiciones, se hipotetiza que: el polo positivo (extraversión) de la dimensión eysenckiana “introversión-extraversión” surgiría como predictor de los CCO de voz (Hipótesis 1); el polo del control emocional de la dimensión eysenckiana "neuroticismo-control” explicaría la ejecución de CCO de ayuda (Hipótesis 2).

Por su parte, la dimensión eysenckiana de Psicoticismo (así como la escala de validez interna de Deseabilidad Social) podrían estar indirectamente relacionadas con los CCO en base a los parámetros comportamentales de los cuales emergen. El Psicoticismo por indicar una marcada predisposición a la frialdad y falta de sentimientos de empatía, difícilmente podría ser un buen predictor de CCO de voz, en tanto que los sujetos no psicóticos estarían más inclinados a involucrarse en tales comportamientos. Finalmente, la Deseabilidad Social, por referirse a la necesidad de mostrar una imagen mejorada de uno mismo, podría emerger como predictor de los CCO de ayuda, aunque difícilmente surgiría como predictor de los CCO de voz. Por lo que se presupone que: el polo más deprimido (ausencia de tendencias psicóticas) de la dimensión eysenckiana “psicoticismo”, explicaría la involucración en CCO de voz (Hipótesis 3); el polo positivo de la escala de validez interna, “deseabilidad social”, surgiría como un predictor de los CCO de ayuda (Hipótesis 4).

\section{Método}

\section{Participantes}

Se estudió una muestra integrada por 335 empleados (195 varones y 140 mujeres) de empresas públicas y privadas argentinas (Tabla 1). El promedio de edad de los sujetos fue de 36 años y el promedio de antigüedad laboral de 3 años. La mayoría de los sujetos tenían formación universitaria o estaban en proceso de completarla. Sólo el 14\% de la muestra ocupaba cargos gerenciales.

Tabla 1

Composición de la muestra en estudio

\begin{tabular}{|c|c|}
\hline Variables consideradas & En porcentajes (\%) \\
\hline \multicolumn{2}{|l|}{ Sexo } \\
\hline Varones & 58 \\
\hline Mujeres & 42 \\
\hline \multicolumn{2}{|l|}{ Educación } \\
\hline Secundaria & 26 \\
\hline Técnica & 10 \\
\hline Universitaria incompleta & 33 \\
\hline Universitaria completa & 31 \\
\hline \multicolumn{2}{|l|}{ Tipo de organización } \\
\hline Pública & 24 \\
\hline Privada & 76 \\
\hline \multicolumn{2}{|l|}{ Cargo } \\
\hline Gerencial & 14 \\
\hline No gerencial & 86 \\
\hline \multicolumn{2}{|l|}{ Tipo de actividad de la organización } \\
\hline Industria & 10 \\
\hline Comercio & 28 \\
\hline Servicios & 32 \\
\hline Educación & 16 \\
\hline Salud & 14 \\
\hline \multirow[t]{2}{*}{$N$} & 335 \\
\hline & Media D.típico \\
\hline Edad & 36,10 \\
\hline Antigüedad laboral & 3,26 \\
\hline
\end{tabular}

\section{Instrumentos}

\section{Hoja de datos sociodemográficos}

La totalidad de la muestra completó una hoja destinada a recabar información acerca de variables tales como edad, sexo, 
nivel de educación alcanzado, tipo de empresa en la que trabaja, cargo que desempeña, antigüedad laboral, etc.

\section{Escalas de Comportamientos de Ciudadanía Organizacional}

Los CCO de ayuda fueron explorados mediante la escala desarrollada por Van Dyne y LePine (1998), integrada por los siguientes 7 ítems: "me ofrezco como voluntario para realizar trabajos que no me exigen que haga”, "oriento a los nuevos empleados que se incorporan a la empresa”, "desempeño funciones que no me son exigidas pero que ayudan a la empresa”, “colaboro con el trabajo de mis compañeros para el éxito de la empresa", "me involucro con la empresa", "ayudo a los demás a aprender sobre la empresa”, "ayudo a mis colegas en sus responsabilidades para con la empresa”. Los CCO de voz fueron evaluados mediante la escala desarrollada por Van Dyne, Graham y Dienesch (1994), integrada por los siguientes 6 ítems: "hago recomendaciones sobre los asuntos que afectan a la empresa", "aliento a mis colegas para que se involucren con los asuntos que conciernen a la empresa", "doy mis opiniones sobre el trabajo aunque sean diferentes y no estén de acuerdo con los demás", "me mantengo informado sobre los asuntos en los que mi opinión puede ser útil a la empresa", "me involucro en asuntos que afectan la calidad de vida en mi trabajo", "doy a conocer mis ideas sobre cambios o nuevos proyectos encarados por mi empresa”. Ambas escalas fueron presentadas bajo un formato Likert de cinco puntos, variando desde "siempre" (5) a "nunca" (1). Análisis de consistencia interna indicaron un Alfa de Cronbach de 0,92 para la escala de ayuda y de 0,94 para la escala de voz (Tabla 2).

\section{Dimensiones eysenckianas de personalidad}

La estructura de la personalidad fue evaluada mediante el Cuestionario de Personalidad de Eysenck (Eysenck \& Eysenck, 1975) en su versión validada para la población argentina por Omar (1988). El cuestionario está integrado por 90 ítems de opción forzada (Si/No), que exploran las tres principales dimensiones eysenckianas de personalidad. La dimensión introversión-extraversión (o factor E), está integrada por 21 ítems (a mayor puntaje, mayor Extraversión); la dimensión neuroticismo-control (o factor N), por 23 ítems (a mayor puntaje, mayor descontrol emocional o Neuroticismo) y la dimensión psicoticismo (o factor P), por 25 ítems (a mayor puntaje, mayor tendencia al Psicoticismo). El EPQ incluye, además, una escala de deseabilidad social (o factor DS) conformada por 21 ítems (a mayor puntaje, mayor predisposición a la DS) que constituye un interesante recurso para detectar a los individuos que tienden a falsear sus respuestas movidos, principalmente, por el deseo de "aparecer mejor". Las cuatro escalas del EPQ presentan índices de consistencia interna muy adecuados, los que varían entre á =0,85 y á = 0,89 (Tabla 2).

\section{Procedimiento}

Luego de obtener la autorización de los directivos de las empresas y la aceptación voluntaria de los sujetos para parti-
Tabla 2

Número de ítems y coeficientes de consistencia interna (Alfa de Cronbach) de las escalas empleadas en el estudio

\begin{tabular}{lcc}
\hline \multicolumn{1}{c}{ Escalas } & Número de ítems & Alfa de Cronbach \\
\hline$E P Q$ & 21 & 0,88 \\
Extraversión & 23 & 0,85 \\
Neuroticismo & 25 & 0,86 \\
Psicoticismo & 21 & 0,89 \\
Deseabilidad social & & \\
\hline CCO & 7 & 0,92 \\
Comportamientos de ayuda & 6 & 0,94 \\
Comportamientos de voz & & \\
\hline
\end{tabular}

cipar del estudio, los empleados respondieron a los cuestionarios de manera individual o en pequeños grupos dentro de su propia empresa, en horarios de trabajo y en lugares físicos especialmente destinados por las autoridades organizacionales para tal propósito. En todos los casos, se garantizó el anonimato y la confidencialidad de la información suministrada, remarcando que la misma sería empleada sólo con fines de investigación científica.

\section{Análisis de los datos}

En primer lugar, y a efectos de controlar el efecto de las variables sociodemográficas sobre la ejecución de los CCO de ayuda y voz, se procedió al cálculo de un análisis de regresión múltiple para la muestra total, en el que los CCO fueron ingresados como variables dependientes y las características sociodemográficas como variables independientes. Procedimiento que es recomendado por Cohen y Cohen (1985), cuando se sospecha que las variables sociodemográficas pueden explicar porciones significativas de la varianza de la variable dependiente, aún cuando se trate de variables dicotómicas. En este caso específico, la existencia de un importante cuerpo de estudios que indican que los CCO son explicados por el sexo y el nivel de escolaridad (Bolino \& Turnley, 2003; Wayne \& Cordeiro, 2003); la edad y la antigüedad laboral (Coyle-Shapiro \& Kessler 2003) y otras factores similares, ameritó el control previo de las variables sociodemográficas de la muestra en estudio.

Seguidamente, y a efectos de probar las hipótesis planteadas, se ejecutó un conjunto de análisis de regresión múltiple por pasos, separadamente para hombres y mujeres (ya que el sexo fue la única variable sociodemográfica que contribuyó significativamente a la explicación de los CCO estudiados). Para la ejecución de tales análisis de regresión, se ingresaron los CCO de voz y de ayuda como variables dependientes y las dimensiones de personalidad (E, N, P y Deseabilidad Social) como variables independientes. El orden de entrada de las variables de personalidad se fundamentó tanto en su relevancia teórica (Eysenck, 1970, 1991), como en la evidencia empírica más reciente. En este sentido, las variables $\mathrm{E}$ y $\mathrm{N}$ se ingresaron en el primer y segundo paso, respectivamente, atendiendo a los hallazgos informados por Barrick, Mount y Judge (2003), Blakely, Andrews y Fuller (2003), Finkelstein y Penner (2004), Fisher (2002) y Van 
Emmerik, Jawahar y Stone (2004). Mientras que las variables $\mathrm{P}$ y DS fueron relegadas al tercer y cuarto paso, respectivamente, porque la evidencia empírica de su rol predictivo de los CCO de voz y ayuda, es prácticamente nula en la bibliografía científica.

\section{Resultados}

El análisis de regresión múltiple calculado con el propósito de determinar si alguna de las variables sociodemográficas consideradas explicaban porciones significativas de la varianza de los CCO de ayuda y de voz (Tabla 3), reveló que el sexo era la única variable demográfica que contribuía significativamente a la explicación de tales comportamientos.

Tabla 3

Análisis de regresión múltiple (variables independientes: datos sociodemográficos; variables dependientes: CCO de ayuda y de voz)

\begin{tabular}{|c|c|c|}
\hline & CCO de Ayuda & CCO de Voz \\
\hline Variables predictoras & $\beta$ & $\beta$ \\
\hline Sexo & $0,769 * *$ & $0,629 * *$ \\
\hline Edad & 0,018 & 0,035 \\
\hline Nivel educacional & 0,116 & $-0,092$ \\
\hline Antigüedad laboral & 0,102 & 0,076 \\
\hline Tipo de organización & 0,009 & 0,076 \\
\hline Cargo desempeñado & 0,105 & 0,098 \\
\hline Actividad de la empresa & 0,064 & 0,087 \\
\hline$R^{2}$ & 0,745 & 0,528 \\
\hline$\Delta R^{2}$ & $0,728 * *$ & $0,497 * *$ \\
\hline$F$ & $43,139 * *$ & $23,216^{*}$ \\
\hline g.l. & $328(7)$ & $328(7)$ \\
\hline
\end{tabular}

$* p<0,05 ; * * p<0,01$

Por lo tanto, con el propósito de verificar las hipótesis planteadas, y dado el singular aporte de la variable sexo en la explicación diferencial de los CCO, se calculó un conjunto de regresiones por pasos (stepwise) separadamente para hombres y mujeres (Tablas 4 y 5), ingresando, en cada paso, las variables de personalidad como predictores y los CCO de ayuda y de voz como variables dependientes (criterios).

En el primer paso, la dimensión Extraversión contribuyó significativamente a la ecuación de regresión, explicando casi el $27 \%\left(\Delta R^{2}=0,269^{* *}\right)$ de la varianza de los CCO de voz. En el paso 2, la dimensión Neuroticismo, contribuyó con un 3,2 \% más a la varianza total $(0,301-0,269=0,032)$, pero el cambio en $R^{2}(0,316-0,295=0,021)$ no alcanzó significación estadística a los niveles convencionales ( $p<0,01$; $p<0,05)$. Cuando se incorporó al modelo la dimensión Psicoticismo en el paso 3, contribuyó con un 6,8\% adicional a la explicación de la varianza total $(0,369-0,301=0,068)$, siendo no significativo el cambio en $R^{2}(0,374-0,316=0,058)$. Finalmente, al incorporar la dimensión de Deseabilidad Social en el cuarto paso, contribuyó con un 4,6\% final a la varianza total $(0,415-$ $0,369=0,046)$, no alcanzando el cambio en $R^{2}(0,438-0,374=$
0,064) significación estadística. Las variables predictoras, en total, explicaron casi un 42\% $(27 \%+3,2 \%+6,8 \%+4,6 \%=$ $41,6 \%$ ) de la varianza total y los valores (y signos) de los coeficientes beta estandarizados (b) indican que la variable Extraversión es el mejor predictor de los CCO de voz entre los empleados varones.

Al considerar el subgrupo de las mujeres se observó que, en el primer paso, la dimensión Extraversión contribuyó significativamente a la ecuación de regresión, explicando el $31,5 \%\left(\Delta R^{2}=0,315^{* *}\right)$ de la varianza. En el paso 2, la dimensión Neuroticismo, contribuyó a la varianza total con un 2,6\% $(0,341$ $-0,315=0,026)$, pero el cambio en $R^{2}(0,382-0,376=0,006)$ no alcanzó significación estadística. Al incorporar al modelo la dimensión de Psicoticismo en el paso 3, se registró una contribución de $13,5 \%$ a la explicación de la varianza total $(0,476-0,341=0,135)$, alcanzando el cambio en $R^{2}(0,538-$ $0,382=0,156)$ significación estadística. En el cuarto paso, al incorporar al modelo explicativo la variable Deseabilidad Social, explicó un 5,3\% más de la varianza total $(0,529-0,476=$ $0,053)$, sin alcanzar el cambio en $R^{2}(0,568-0,538=0,030)$ adecuada significación estadística. Las variables predictoras, en total, explicaron casi un 53\% (31,5\% + 2,6\% $+13,5 \%+5,3 \%=52,9 \%)$ de la varianza total y los valores $(\mathrm{y}$ signos) de los coeficientes beta estandarizados indican que la Extraversión y la tendencia a comportamientos no psicóticos, son los mejores predictores de la involucración de CCO de voz por parte de las mujeres en sus lugares de trabajo. Los resultados obtenidos dieron total apoyo a la hipótesis 1 que postulaba que la Extraversión predeciría los CCO de voz, desde el momento en que la E presentó efectos significativos sobre la ejecución de los CCO de voz tanto entre los varones como entre las mujeres. A su vez, los resultados dieron apoyo parcial a la hipótesis 3, que postulaba que el polo más deprimido (ausencia de tendencias psicóticas) de la dimensión Psicoticismo, explicaría la ejecución de CCO de voz, ya que esta circunstancia sólo se verificó en el subgrupo de las mujeres.

Seguidamente, y con idéntica metodología, se procedió a la verificación de las hipótesis inherentes a la predicción de los CCO de ayuda por parte de las dimensiones eysenckianas de personalidad.

Los resultados obtenidos (Tabla 5) indican que la dimensión Neuroticismo es la única variable de personalidad que predice los comportamientos de ayuda en el ámbito laboral, tanto entre los varones, como entre las mujeres, al explicar una importante porción de la varianza de tales comportamientos (variando entre el $24 \%$ para los varones y el 22,9\% para las mujeres). Los signos negativos que preceden a los correspondientes coeficientes beta, indican que el aporte predictivo al modelo explicativo proviene del polo más deprimido de la variable neuroticismo-control (o sea el polo del control emocional), tal como fuera hipotetizado, por lo que los resultados confirman plenamente la hipótesis 2. Por otro lado, y contrariamente a lo planteado en la hipótesis 4, la tendencia a mostrar una imagen mejorada de uno mismo (factor Deseabilidad Social), si bien explica cerca del 10\% de la 
Tabla 4

Regresión múltiple por pasos (stepwise) considerando como variables independientes las dimensiones de personalidad y como variables dependientes los CCO de voz, separadamente para varones $(n=195)$ y mujeres $(n=140)$

\begin{tabular}{|c|c|c|c|c|}
\hline \multirow[b]{2}{*}{ Variables predictoras } & \multicolumn{4}{|c|}{ Variable Dependiente: CCO de voz } \\
\hline & Paso 1 & Paso 2 & Paso 3 & Paso 4 \\
\hline Varones $(\mathrm{n}=195)$ & $\beta$ & $\beta$ & $\beta$ & $\beta$ \\
\hline $\begin{array}{l}\text { Extraversión } \\
\text { Neuroticismo } \\
\text { Psicoticismo } \\
\text { Deseabilidad Social }\end{array}$ & $0,278^{*}$ & $\begin{array}{c}0,321^{* *} \\
-0,066\end{array}$ & $\begin{array}{c}0,356^{* *} \\
-0,072 \\
0,129 *\end{array}$ & $\begin{array}{c}0,408^{* *} \\
-0,077 \\
0,099 \\
0,068 \\
\end{array}$ \\
\hline $\begin{array}{r}R^{2} \\
\Delta R^{2} \\
\text { Cambio en } R^{2} \\
F \\
\text { g.l. }\end{array}$ & $\begin{array}{c}0,295 \\
0,269 * * \\
0,295^{* *} \\
12,76 \\
194(1)\end{array}$ & $\begin{array}{c}0,316 \\
0,301^{* *} \\
0,021 \\
31,73 \\
193(2)\end{array}$ & $\begin{array}{c}0,374 \\
0,369 * * \\
0,058 \\
54,82 \\
192(3)\end{array}$ & $\begin{array}{c}0,438 \\
0,415^{* *} \\
0,064 \\
76,24 \\
191(4)\end{array}$ \\
\hline Mujeres $(n=140)$ & $\bar{\beta}$ & $\beta$ & $\beta$ & $\beta$ \\
\hline $\begin{array}{l}\text { Extraversión } \\
\text { Neuroticismo } \\
\text { Psicoticismo } \\
\text { Deseabilidad Social }\end{array}$ & $0,425^{* *}$ & $\begin{array}{l}0,478 * * \\
-0,079\end{array}$ & $\begin{array}{c}0,567^{* *} \\
-0,058 \\
-0,156^{*} \\
0,144^{*}\end{array}$ & $\begin{array}{c}0,619 * * \\
-0,016 \\
-0,178^{*} \\
0,089\end{array}$ \\
\hline $\begin{array}{r}R^{2} \\
\Delta R^{2} \\
\text { Cambio en } R^{2} \\
F \\
\text { g.l. }\end{array}$ & $\begin{array}{c}0,376 \\
0,315^{* *} \\
0,376^{* *} \\
140,55 \\
139(1)\end{array}$ & $\begin{array}{c}0,382 \\
0,341 * * \\
0,006 \\
90,47 \\
138(2)\end{array}$ & $\begin{array}{c}0,538 \\
0,476^{* *} \\
0,156^{*} \\
165,63 \\
137(3)\end{array}$ & $\begin{array}{c}0,568 \\
0,529 * * \\
0,053 \\
163,88 \\
136(4)\end{array}$ \\
\hline
\end{tabular}

$* p<0,05 ; * * p<0,01$

Tabla 5

Regresión multiple por pasos (stepwise) considerando como variables independientes (predictores) las dimensiones de personalidad y como variables dependientes los CCO de ayuda, separadamente para varones $(n=195)$ y mujeres $(n=140)$

\begin{tabular}{|c|c|c|c|c|}
\hline \multirow[b]{2}{*}{ Variables predictoras } & \multicolumn{4}{|c|}{ Variable Dependiente: CCO de voz } \\
\hline & Paso 1 & Paso 2 & Paso 3 & Paso 4 \\
\hline Varones $(\mathrm{n}=195)$ & $\beta$ & $\beta$ & $\beta$ & $\beta$ \\
\hline $\begin{array}{l}\text { Extraversión } \\
\text { Neuroticismo } \\
\text { Psicoticismo } \\
\text { Deseabilidad Social }\end{array}$ & 0,104 & $\begin{array}{c}0,107 \\
-0,312 * *\end{array}$ & $\begin{array}{l}-0,102 \\
-0,415^{* *} \\
0,078\end{array}$ & $\begin{array}{c}0,094 \\
-0,548^{*} \\
-0,056 \\
0,471\end{array}$ \\
\hline $\begin{array}{r}R^{2} \\
\Delta R^{2} \\
\text { Cambio en } R^{2} \\
F \\
\text { g.l. }\end{array}$ & $\begin{array}{c}0,078 \\
0,057^{* *} \\
0,078^{* *} \\
1,23 \\
194(1)\end{array}$ & $\begin{array}{c}0,301 \\
0,297 * * \\
0,240 \\
19,35 \\
193(2)\end{array}$ & $\begin{array}{c}0,378 \\
0,308^{* *} \\
0,077 \\
22,18 \\
192(3)\end{array}$ & $\begin{array}{c}0,426 \\
0,407 * * \\
0,048 \\
37,40 \\
191(4)\end{array}$ \\
\hline Mujeres $(\mathrm{n}=140)$ & $\beta$ & $\beta$ & $\beta$ & $\beta$ \\
\hline $\begin{array}{l}\text { Extraversión } \\
\text { Neuroticismo } \\
\text { Psicoticismo } \\
\text { Deseabilidad Social }\end{array}$ & 0,099 & $\begin{array}{l}0,109 \\
-0,456\end{array}$ & $\begin{array}{r}0,110 \\
-0,478 \\
-0,100\end{array}$ & $\begin{array}{r}0,113 \\
-0,748 \\
-0,057 \\
0,654 \\
\end{array}$ \\
\hline $\begin{array}{r}R^{2} \\
\Delta R^{2} \\
\text { Cambio en } R^{2} \\
F \\
\text { g.l. }\end{array}$ & $\begin{array}{c}0,099 \\
0,097 \\
0,099 \\
1,55 \\
139(1)\end{array}$ & $\begin{array}{c}0,376 \\
0,326^{* *} \\
0,277 \\
40,47 \\
138(2)\end{array}$ & $\begin{array}{c}0,424 \\
0,401^{* *} \\
0,048 \\
16,53 \\
137(3)\end{array}$ & $\begin{array}{c}0,512 \\
0,497 * * \\
0,088 \\
76,48 \\
136(4)\end{array}$ \\
\hline
\end{tabular}

$* p<0,05 ; * * p<0,01$ 
varianza total en ambos modelos (varones y mujeres), no llega a emerger como un predictor idóneo de los CCO de ayuda, ya que los cambios en $R^{2}$ no alcanzan valores significativos.

\section{Conclusiones}

Los resultados obtenidos indican que la ejecución de CCO, especialmente aquellos orientados a ayudar al supervisor y/o compañeros de trabajo, y los vinculados a manifestar las opiniones personales vinculadas con diferentes aspectos de la empresa o institución, pueden predecirse a partir de determinados rasgos o características de personalidad. En armonía con los hallazgos de Finkelstein y Penner (2004), Fisher (2002) y Van Emmerik, Jawahar y Stone (2004), la estabilidad emocional surge como el predictor más fuerte de los CCO de ayuda desplegados tanto por varones como por mujeres en su ámbito laboral. Posiblemente, y tal como lo puntualizara Diedendorff y sus colegas (2002), habría entornos de trabajo (aquellos exentos de altas demandas) que potenciarían las predisposiciones hacia los afectos positivos, propiciando el despliegue de comportamientos prosociales dentro del equipo laboral. En sintonía con los resultados recientemente informados por Baruch, O’Creevy, Hind y Vigoda-Gadot (2004) y Barrick, Mount y Judge (2003), de la presente verificación empírica también surgen claras evidencias de la capacidad de la dimensión eysenckiana de extraversión para predecir comportamientos de ciudadanía vinculados con la intención manifiesta de exponer puntos de vistas, opiniones y/o sugerencias que contribuyan al mejoramiento continuo de la organización. La posibilidad de dar a conocer ideas, informaciones y opiniones para mejorar tanto su propio trabajo como el de la empresa (CCO de voz), se erige como un patrimonio de los sujetos conversadores, sociables y expansivos, en abierta oposición a los sujetos ubicados en el sector más deprimido de la dimensión temperamental, los introvertidos, quienes por la timidez, retraimiento e inseguridad que los caracteriza preferirían permanecer en silencio sin dar a conocer sus opiniones. Hablar o callar surgen, por lo tanto, como dos polos opuestos. Tan opuestos como los polos extraversión-introversión de la dimensión temperamental eysenckiana. Para Van Dyne, Ang y Botero (2003), tanto el silencio como la voz tienen consecuencias diferenciales sobre el trabajo organizacional. Postulan que el silencio es más ambiguo que la voz ya que hay más probabilidades de hacer atribuciones erróneas de los motivos del silencio, que los de la voz y que las atribuciones erróneas del silencio podrían llevar a consecuencias incongruentes (tanto positivas como negativas) para los empleados. Por lo que proponen que tanto la voz como el silencio deberían ser conceptualizados en forma separada, como constructos multidimensionales, ya que el silencio no constituye simplemente la ausencia de voz, sino que muchas veces es la manifestación del ocultamiento de información importante. Desde la perspectiva de Bowen y Blackmon (2003), los CCO de voz son significativamente influenciados por la percepción individual de las actitudes hacia un determinado asunto dentro del grupo de trabajo. Sostienen que los individuos se mostrarán más propensos a hablar cuando crean que su posición será sostenida por los demás, en caso contrario, permanecerán en silencio. Explican tales suposiciones en base a "la espiral del silencio", la que enfatiza sobre la presión horizontal que ejerce la amenaza y el correspondiente miedo al aislamiento sobre la gente que expresa sus opiniones de manera abierta y honesta.

Si bien la evidencia reunida no alcanza a dar pleno respaldo a las hipotetizadas relaciones entre CCO de voz y ausencia de tendencias psicóticas, surgen indicios de la mayor predisposición de las mujeres no psicóticas a involucrarse con mayor frecuencia en tales comportamientos. Hallazgo que coincide con los resultados del estudio de LePine, Erez y Johnson (2002), cuando señalan que los sujetos ecuánimes, empáticos y sin predisposiciones a los comportamientos transgresores (características propias de los no psicóticos, Omar, 2002) son los más predispuestos a involucrarse en CCO de voz. Son las mujeres las que también aparecen como más proclives a comprometerse en CCO de ayuda, tal como se desprende de los valores de la Tabla 5. La mayor capacidad predictiva del sexo femenino sobre la ejecución de CCO de ayuda y voz, es consistente con observaciones previas (Bolino \& Turnley, 2003; Wayne \& Cordeiro, 2003), que remarcan la mayor tendencia de las mujeres (quizá por que las características de tales CCO son consistentes con cualidades estereotípicamente adscriptas al género femenino) a comprometerse en comportamientos prosociales, dedicar más tiempo a exponer sus puntos de vista y brindar una ayuda de mayor calidad, que los hombres.

Al iniciarse el nuevo milenio es apropiado preguntarse cuánto hemos aprendido acerca de las relaciones entre personalidad y comportamientos organizacionales durante el pasado siglo y embarcar nuestras investigaciones hacia nuevas direcciones. Los resultados obtenidos indican que la nueva agenda para las futuras investigaciones debería incluir, al menos: (1) el análisis de los comportamientos que se van agregando gradualmente a la red nomológica de los CCO (como es el caso del silencio, sobre todo para poder crear la alternativa virtuosa a la denominada espiral del silencio y que todos tengan la posibilidad de hacer escuchar su voz organizacional); (2) la consideración del rol de los valores éticos, sean individuales u organizacionales, como predictores de los CCO o síndrome del buen soldado - ya que, como lo señala Turnipseed (2002), los CCO tienen un fuerte componente ético, desde el momento en que pueden ser morales o inmorales - y pueden ser, simplemente, la manifestación de comportamientos éticos en el lugar de trabajo; (3) la exploración de otros constructos de personalidad, tal como el de personalidad "resistente", recientemente introducido por Turnipseed (2003), referido a la capacidad de proteger al sujeto de la enfermedad producida por el estrés laboral y, sobre todo, (4) el tipo de prácticas organizacionales que fomenten y sustenten la ejecución de comportamientos que van más allá de los requerimientos del cargo y puedan transformarse en un elemento clave para el éxito organizacional, como lo son los comportamientos de ciudadanía. 


\section{Referencias}

Aryee, S., Budhwar, P. S., \& Chen, Z. X. (2002). Trust as a mediator of the relationship between organizational justice and work outcomes: test of a social exchange model. Journal of Organizational Behavior, 23, 267285.

Barrick, M. R., Mount, M. K., \& Judge, T. A. (2003). Personality and performance at the beginning of the new millennium: what do we know and where do we go next? Journal of Applied Psychology, 52, 201-216.

Baruch, Y., O’Creevy, M., Hind, P., \& Vigoda-Gadot, E. (2004). Pro social behavior and job performance: does the need for control and the need for achievement make a difference? Social Behavior \& Personality, 32, 399411.

Bateman, T. S., \& Organ, D. W. (1983). Job satisfaction and the good soldier: the relationship between affect and "citizenship". Academy of Management Journal, 26, 587-595.

Becker, T. E., \& Kernan, M. C. (2003). Matching commitment to supervisors and organizations to in-role and extra-role performance. Human Performance, 16, 327-348.

Blakely, G. L., Andrews, M. C., \& Fuller, J. (2003). Are chameleons good citizens? A longitudinal study of the relationship between self-monitoring and organizational citizenship behavior. Journal of Business \& Psychology, $18,131-144$.

Bolino, M. C., \& Turnley, W. H. (2003). Going the extra mile: cultivating and managing employee citizenship behavior. Academy of Management Executive, 17, 60-71.

Bowen, F., \& Blackmon, K. (2003). Spirals of silence: the dynamic effects of diversity on organizational voice. Journal of Management Studies, 40, 1393-1417.

Cohen, J., \& Cohen, P. (1983). Applied multiple regression/correlation analysis for the behavioral sciences. Hillsdale, New Jersey: Erlbaum.

Coyle-Shapiro, J. A. M., \& Kessler, I. (2003). The employment relationship in the UK public sector: a psychological contract perspective. Journal of Public Administration Research \& Theory, 13, 213-230.

Diefendorff, J. M., Brown, D. J., Kamin, A. M., \& Lord, R. G. (2002). Examining the roles of job involvement and work centrality in predicting organizational citizenship behaviors and job performance. Journal of Organizational Behavior, 23, 93-108.

Ehrhart, M. G. (2004). Leadership and procedural justice climate as antecedents of unit-level organizational citizenship behavior. Personnel Psychology, 57, 61-94.

Eysenck, H. J., \& Eysenck S. B. G. (1975). Manual of the Eysenck Personality Questionnaire. Londres: Hodder \& Stoughton.

Eysenck, H. J., \& Eysenck, M. W. (1985). Personality and individual difference. a natural science approach. Nova York: Plenum.

Eysenck, H. J. (1970). Fundamentos biológicos de la personalidad. Barcelona: Fontanella.

Eysenck, H. J. (1991). Dimensions of personality: 16, 5 or 3? Criteria for a taxonomic paradigm. Personality and Individual Differences, 12, 773-790.

Finkelstein, M. A., \& Penner, L. A. (2004). Predicting organizational citizenship behavior: integrating the functional and role identity approaches. Social Behavior \& Personality, 32, 383-398.

Fisher, C. D. (2002). Antecedents and consequences of real time affective reactions at work. Motivation \& Emotion, 26, 3-30.

George, J. M., \& Brief, A. P. (1992). Feeling good-doing good: a conceptual analysis of the mood at work-organizational spontaneity relationship. Psychological Bulletin, 112, 310-329.

George, J. M. (1991). State of trait: effects of positive mood on pro social behaviors at work. Journal of Applied Psychology, 76, 299-307.
Hui, C., Lee, C., \& Rousseau, D. M. (2004). Employment relationships in China: do workers relate to the organization or to people? Organization Science, 15, 232-240.

Kickul J., Lester, S. W., \& Finkl, J. (2002). Promise breaking during radical organizational change: do justice interventions make a difference? Journal of Organizational Behavior, 23, 469-488.

Konovsky, M. A., \& Organ, D. W. (1996). Dispositional and contextual determinants or organizational citizenship behavior. Journal of Organizational Behavior, 17, 253-266.

LePine, J. A., \& Van Dyne, L. (1998). Predicting voice behavior in work groups. Journal of Applied Psychology, 83, 853-868.

LePine, J. A., Erez, A., \& Johnson, D. E. (2002). The nature and dimensionality of organizational citizenship behavior: a critical review and meta-analysis. Journal of Applied Psychology, 87, 52-65.

MacKenzie, S. B., Podsakoff, P. M., \& Fetter, R. (1993). The impact of organizational citizenship behavior evaluation of sales person performance. Journal of Marketing, 57, 70-80.

Mischel, W. (1977). The interaction of person and situation. In I. D. Magnusson \& N. S. Endler (Orgs.), Personality at the cross roads: current issues in international psychology (pp. 235-238). Hillsdale, New Jersey: Erlbaum.

Morrison, E. W. (1994). Role definitions and organizational citizenship behavior: the importance of the employee's perspective. Academy of Management Journal, 37(6), 1543-1567.

Motowidlo, S. J., \& Van Scotter, R. (1994). Evidence that task performance should be distinguished from contextual performance. Journal of Applied psychology, 79, 475-480.

Omar, A. (1988). Estandarización argentina de los cuestionarios de personalidad de Eysenck. Revista Chilena de Neuro-Psiquiatría, 42, 83-95.

Omar, A. (2002). Búsqueda de sensaciones e intensidad de afecto. In M. Hernández González (Org.), Motivación animal y humana (pp. 317-327). México: El Manual Moderno.

Organ, D. W., \& Konovski. M. A. (1989). Cognitive versus determinants of organizational citizenship behavior. Journal of Applied Psychology, 74, 157-164.

Organ, D. W., \& Ryan, K. (1995). A meta-analytic review of attitudinal and dispositional predictors of organizational citizenship behavior. Personnel Psychology, 48, 775-802.

Organ, D. W. (1988). Organizational citizenship behavior: the good soldier syndrome. Lexington, Massachusetts: Lexington Books.

Organ, D. W. (1994). Personality and organizational behavior. Journal of Management, 20, 465-478.

Pearce, C. L., \& Herbik, P. A. (2004). Citizenship behavior at the team level of analysis: the effects of team leadership, team commitment, perceived team support, and team size. Journal of Social Psychology, 144, 293-310.

Podsakoff, P. M., MacKenzie, S. B., \& Hui, C. (1993). Organizational citizenship behavior and managerial evolutions of employee performance: a review and suggestions for future research. Research in Personnel and Human Resource Management, 11, 1-40.

Porto, J. B., \& Tamayo, A. (2003). Desenvolvimento e validação da Escala de Civismo nas Organizações. Estudos de Psicologia, 8(3), 393-402. Versão eletrônica obtida, em 10/outubro/2004, do website http://www.scielo.br/.

Puffer, S. M. (1987). Pro social behavior, non compliant behavior, and work performance among commission sales people. Journal of Applied Psychology, 72, 615-621.

Rego, A. (2000). Justiça y comportamientos de ciudadanía en las organizaciones: uma abordagem sem tabus. Lisboa: Sílabo.

Siqueira, M. M. M., Gomide Jr., S., \& Oliveira, A. F. (2002). Cidadania, justiça e cultura nas organizações. São Bernardo do Campo: UMESP. 
Smith, C. A., Organ, D. W., \& Near, J. P. (1983). Organizational citizenship behavior: its nature and antecedents. Journal of Applied Psychology, 68, 653-663.

Staw, B. M., Bell, N. E., \& Clausen, J. A. (1986). The dispositional approach to job attitudes: a life time longitudinal test. Administrative Science Quarterly, 31, 56-77.

Tamayo, A. (1998). Prioridades axiológicas, tempo de serviço e cidadania organizacional. Psicologia: Teoria e Pesquisa, 14, 35-40.

Turnipseed, D. L. (2002). Are good soldiers good? Exploring the link between organization citizenship behavior and personal ethics. Journal of Business Research, 55, 1-15.

Turnipseed, D. L. (2003). Hardy personality: a potential link with organizational citizenship behavior. Psychological Reports, 93, 529-543.

Van Dyne, L., \& LePine, J. A. (1998). Helping and voice extra-role behaviors: evidence of construct and predictive validity. Academy of Management Journal, 41, 108-119.

Van Dyne, L., \& Pierce, J. L. (2004). Psychological ownership and feelings of possession: three field studies predicting employee attitudes and organizational citizenship behavior. Journal of Organizational Behavior, 25, 439-459.

Van Dyne, L., Ang, S., \& Botero, I. C. (2003). Conceptualizing employee silence and employee voice as multidimensional constructs. Journal of Management Sales, 40, 1359-1392.
Van Dyne, L., Cummings, L. L. \& Parks, J. M. (1995). Extra-role behaviors: in pursuit of construct and definitional clarity (A bridge of muddied waters). Research in Organizational Behavior, 17, 215285.

Van Dyne, L., Graham, J. W., \& Dienesch, R.M. (1994). Organizational citizenship behavior: construct redefinition, operationalization, and validation. Academy of Management Journal, 37, 765-802.

Van Dyne, L., Vandewalle, D., Kostova, T., Latham, M. E., \& Cummings, L. L. (2000). Collectivism, propensity to trust and self-esteem as predictors of organizational citizenship in a non-work setting. Journal of Organizational Behavior, 21, 3-23.

Van Emmerik, I. H., Jawahar, I. M., \& Stone, T. H. (2004). The relationship between personality and discretionary helping behaviors. Psychological Reports, 95, 355-365.

Vey, M. A., \& Campbell, J. P. (2004). In-role or extra-role organizational citizenship behavior: which are we measuring? Human Performance, 17, 119-135.

Wayne, J. H., \& Cordeiro, B. L. (2003). Who is a good organizational citizen? Social perception of male and female employees who use family leave. Sex Roles, 49, 233-246.

Yoon, M. H., \& Suh, J. (2003). Organizational citizenship behaviors and service quality as external effectiveness of contact employees. Journal of Business Research, 56, 597-611.

Alicia Omar, doctora en Psicología por la Universidad Nacional de San Luís (Argentina), es investigadora en el Consejo Nacional de Investigaciones Científicas y Técnicas (CONICET), Argentina. Dirección: Italia 1365, $1^{0}$ A; 2000 Rosario, Argentina. Tel: (54) (0341) 4480314, Fax (54) (0341) 4480314. E-mail: agomar@arnet.com.ar

Hugo Uribe Delgado, master en Sociología por Instituto de Sociología, Academia de Ciencias de la República Checa, es investigador en el Instituto de Investigaciones de la Universidad Nacional de Rosario, Argentina. Email: iinvest@fhumyar.unr.edu.ar 